\title{
Time-Resolving Quantum Measurement Enables Energy-Efficient, Large-Alphabet Communication
}

\author{
I.A. Burenkov ${ }^{1,2,{ }^{*}}{ }^{*}$ M.V. Jabir, ${ }^{2}$ A. Battou, ${ }^{2}$ and S.V. Polyakov ${ }^{2,3, \dagger}$ \\ ${ }^{1}$ Department of Physics, Joint Quantum Institute, University of Maryland, College Park, Maryland 20742, USA \\ ${ }^{2}$ National Institute of Standards and Technology, Gaithersburg, Maryland 20899, USA \\ ${ }^{3}$ Department of Physics, University of Maryland, College Park, Maryland 20742, USA
}

(Received 27 November 2019; accepted 28 August 2020; published 21 September 2020)

\begin{abstract}
Information exchange requires a measurement of physical states. Because quantum measurements enable accuracy beyond the classical shot-noise limit, they are successfully used to develop measurement tools and applications. In particular, quantum-measurement-enhanced strategies are used for the discrimination of nonorthogonal coherent states. The efficient discrimination of these states is crucial for optical communication networks, that are now approaching the classical limits of the underlying physical systems. However, quantum-enhanced discrimination strategies demonstrated to date are based on legacy communication protocols designed for classical measurements and thus provide only a limited advantage. In our work, we use photon detection times readily available in quantum measurement, but not accessible by classical means. We measure and use these times to maximize our knowledge about faint nonorthogonal coherent states. We employ communication strategies designed to benefit most from this knowledge. This holistic approach in our experiment has resulted in the record low error rates in discrimination of multiple faint nonorthogonal coherent states carrying energy corresponding to just one photon per bit of transmitted information. We demonstrate successful discrimination of large alphabets of optical states $(4 \leq M \leq 16)$ beyond the ideal classical shot-noise limit, showing the scalability of quantum-measurement-enabled communication. This experimental work explores unforeseen advantages of quantum measurement on one hand, and may help address the capacity crunch in modern optical networks caused by the exponential growth of data exchange on the other.
\end{abstract}

DOI: 10.1103/PRXQuantum.1.010308

\section{INTRODUCTION}

The connection between information and physics is now well established as a result of the progress in information theory and computer science on one hand [1,2], and quantum physics on the other [3]. The physical embodiment of information makes communication an inherently physical measurement problem. Because quantum measurements can greatly surpass the capabilities of classical measurement, quantum-enhanced communication has resulted in many research efforts [4-13]. It is remarkable that quantum measurement provides an advantage even when using communication protocols originally developed for classical measurement methods and maintains that advantage

\footnotetext{
*ivan.burenkov@gmail.com

†spoly@nist.gov

Published by the American Physical Society under the terms of the Creative Commons Attribution 4.0 International license. Further distribution of this work must maintain attribution to the author(s) and the published article's title, journal citation, and DOI.
}

even in the presence of a significant loss, which is common to all communication channels [14-17]. Because, generally speaking, the properties of quantum measurements differ significantly from those of classical measurements, the advantages offered by quantum measurements cannot be fully exploited with such legacy protocols. To unleash the full potential of quantum measurement, the communication protocol and matching receiver should be designed together, taking into account their quantum properties.

Coherent states are ideal for practical long-distance communications because they are easy to prepare and manipulate and their statistical properties are resilient to losses. Using an alphabet of multiple coherent states to encode information can increase the capacity of a communication channel. Therefore, $M$-ary alphabets are often used in practical communication links. While it is common for a classical link to use very long alphabets, currently there are no experimental demonstrations of quantum receivers beyond $M=4$. Furthermore, legacy adaptive quantum receivers based on single-photon or photonnumber-resolving detection in predetermined time intervals $[14-16]$ are not optimal $[13,18]$. The use of photon 
arrival times for receivers was theoretically proposed by Bondurant in Ref. [6], with cyclic probing strategy at the receiver. In Ref. [13] it was found that photon detection times could be used more efficiently if a Bayesian probing strategy is employed. In Ref. [18], we introduced a class of communication protocols that take extra advantage of the detection times and the Bayesian probing strategy, i.e. the class of protocols designed to optimize the quantum measurement. The matching of the modulation protocol and the receiver is particularly advantageous for discrimination of the nonorthogonal coherent states with a low average number of photons and a large number of states $M$ in communication alphabets.

Here we experimentally demonstrate a quantuminspired communication link and achieve the highest energy sensitivity to date with extremely faint optical signals. Energy sensitivity is defined here as the inverse of the error rate at the receiver for a given energy of an optical input (smaller error rate means higher sensitivity). Paired with our communication protocol [18], the time-resolving receiver exhibits a favorable scaling versus $M$ of the energy sensitivity for a given energy of the input coherent state. We report a record energy sensitivity for input optical energy at the receiver of approximately $3 \times 10^{-19} \mathrm{~J} / \mathrm{bit}$ (approximately 1 photon per bit on average) for a range of alphabet lengths: $4 \leq M \leq 16$. We achieve unconditional (that is, as compared to detection efficiency of unity) discrimination of $M$ nonorthogonal coherent states below the classical uncertainty bound: the shot-noise limit (SNL) of an optimal classical receiver [19].

Our results demonstrate that the unique properties of quantum measurement can improve classical communication in a conceptual way. Here, photon detection times are used to enable quantum state discrimination experiments beyond the sensitivity of quantum receivers demonstrated to date.

\section{THE MODULATION SCHEME}

To maximize the advantage of quantum measurements we implement a family of $M$-ary coherent frequency shift keying (CFSK) modulation schemes. The CFSK uses $M$ phase-synchronized top-hat coherent states that differ in initial phases and frequencies such that detunings and initial phases between the adjacent states are equal [18]. Each pulse is a coherent state $|\psi\rangle=\left|\alpha\left(\omega_{s}, \theta_{s}\right)\right\rangle=\mid \alpha\left[\omega_{0}+\right.$ $(s-1) \Delta \omega,(s-1) \Delta \theta]\rangle, s \in 1 . . M$, where the separation between adjacent symbols in frequency is $\Delta \omega$ and the initial phase separation is $\Delta \theta$. The detuning $\Delta \omega$ is normalized by the optical bandwidth of the signal pulses $1 / T$, therefore $\Delta \omega T=2 \pi$ corresponds to the minimal detuning of classically orthogonal states. Thus, our protocol has two independent optimization parameters: $\Delta \theta$ and $\Delta \omega T$. Here we experimentally investigate the case of the frequency shifts $0<\Delta \omega T<2 \pi$, i.e., smaller than the bandwidth of optical signal pulses. By adapting these parameters to the properties of quantum measurement employed at the receiver, one can improve the energy and/or bandwidth efficiency of a communication channel. As a result, our modulation scheme improves the energy sensitivity of legacy nonorthogonal classical protocols (such as PSK) while using less channel bandwidth than orthogonal classical modulation protocols (such as OFSK and PPM). It also results in lower error probabilities and lower theoretical bounds in comparison to orthogonal modulations [19, $20]$ when properly optimized and paired with a quantum receiver [18].

We point out that this modulation scheme can also be adapted to experimental conditions, i.e., nonideal visibility of displacement, dark counts at the detector, etc. The CFSK classical sensitivity limit is the SNL of the optimal classical receiver, see Appendix $\mathrm{C}$ for derivation. The Helstrom bound for nonorthogonal CFSK states is derived elsewhere (see the Supplemental Material of Ref. [18]). Unlike legacy modulation schemes developed for classical receivers this modulation scheme enables the optimization of a quantum-enhanced communication channel.

\section{THE TIME-RESOLVED QUANTUM RECEIVER}

The CFSK quantum receiver employs an adaptive displacement followed by a time-resolved single-photon detection, (Fig. 1). The receiver performs a quantum measurement by testing if the hypothesized state $\left|\beta_{h}\right\rangle=$ $\left|\beta\left(\omega_{h}, \theta_{h}\right)\right\rangle$ corresponds to the state of the input field $\left|\psi_{s}\right\rangle$. If the hypothesis is correct, and the local oscillator (LO) is balanced with the signal, i.e., $|\alpha|^{2}=|\beta|^{2}$, the input field is displaced to the vacuum and no photons can be detected at the output. In this ideal case, if even a single photon is detected, the hypothesis fails. The most likely hypothesis is updated after every photon detection. Then, at $t=T$, the current most likely hypothesis represents our best knowledge of the input state $s$. A time-resolved receiver was theoretically proposed by Bondurant [6] in 1993. In that seminal work, hypotheses are probed in a cyclic order and a photon detection is used as a trigger for a hypothesis change. In a recent work [13], it was theoretically shown that photon detection times can be used to find the most probable state of the input field via Bayesian inference. This Bayesian strategy significantly improves the energy sensitivity of a quantum receiver. Therefore, in contrast to a Bondurant receiver where a single-photon detection excludes the initial hypothesis only, the receiver using Bayesian inference extracts and uses this additional temporal information to displace the input state to the most probable state. Because the CFSK states differ in frequency, the displacement of the input that is based on an 


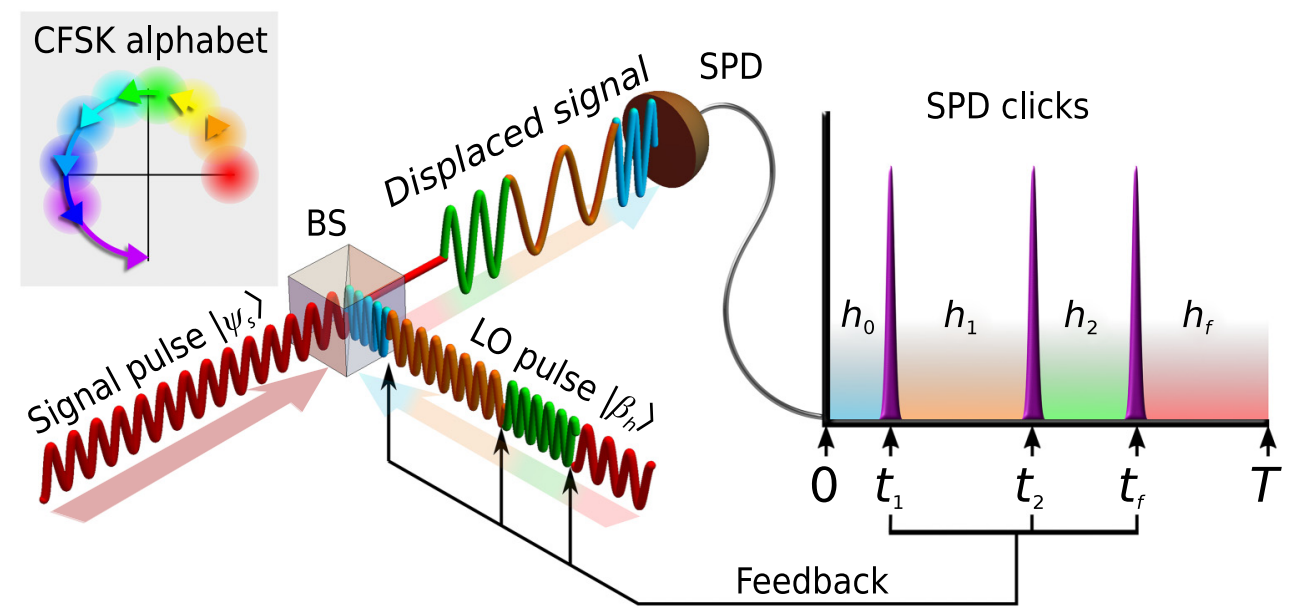

FIG. 1. Time-resolving quantum receiver. The signal pulse $\left|\psi_{s}\right\rangle$ is displaced at a 99:1 beam splitter (BS) with the adaptive local oscillator $\left|\beta_{h}\right\rangle$, whose parameters depend on the hypothesized most probable input state $h$ and followed with a single-photon detector (SPD). Colors represent frequency detunings. The displaced signal for the correct hypothesis nulls the output, whereas an incorrect hypothesis results in a temporal fringe with the probability density of a photon detection varying in time, uniquely for each pair of $\left|\psi_{s}\right\rangle$ and $\left|\beta_{h}\right\rangle$. Each detection time $t_{k}, k \in 1 . . f$, is used to find the posterior hypothesis $h_{k}$. Inset: an $M=8$ CFSK alphabet schematically depicted in a complex phase diagram starting at the beginning of a symbol pulse. In the rotating frame of the first state, other states revolve around the origin at different rates, represented by the length of arrows.

incorrect hypothesis results in a fringe in time, whose temporal features differ for different signal-LO pairs. Therefore, the probability density to detect a photon varies in time, and, consequently, the photon detection times contain information about the input state.

In this work, we experimentally implement a timeresolved quantum receiver and demonstrate its below shotnoise performance. The receiver calculates the Bayesian probabilities of all $M$ possible alphabet states to be the input state, $\zeta_{t}(m), m=1 . . M$. Upon each photon detection at $t_{k}, k \geq 1$, the alphabet state with the highest Bayesian probability $\zeta_{t_{k}}(m)$ is used as a new hypothesis $h_{k}$. The LO is adjusted to $\left|\beta_{h_{k}}\right\rangle$ to displace the most probable state to vacuum. Before the first photon is detected, the a priori probabilities are equal for all states, $\zeta_{0}=1 / M$ at $t_{0}=0$. Simulations show that the final error rate does not depend on the initial hypothesis, so $h_{0}$ can be random. The Bayes' rule to recursively calculate a posteriori probabilities $\zeta_{t_{k}}(m)$ based on a priori probabilities $\zeta_{t_{k-1}}(m)$ is

$$
\zeta_{t_{k}}(m)=\frac{\aleph\left(t_{k}, t_{k-1}, m, h_{k-1}\right) \zeta_{t_{k-1}}(m)}{\sum_{j=1}^{M} \aleph\left(t_{k}, t_{k-1}, j, h_{k-1}\right) \zeta_{t_{k-1}}(j)} .
$$

The Bayesian likelihood $\aleph\left(t_{k}, t_{k-1}, m, h_{k-1}\right)$ that the signal input state is $m$, is the probability density to detect a photon at $t_{k}$ if the previous photon is detected at $t_{k-1}$ and the displacement is $h_{k-1}$. Because the input and the LO are coherent states, the field at the detector is a modulated coherent state described by a nonhomogeneous Poisson process [21]

$$
\begin{aligned}
& \aleph\left(t_{k}, t_{k-1}, m, h_{k-1}\right)= \\
& \quad\left[\left\langle n\left(m, h_{k-1}, t_{k}\right)\right\rangle / T\right] e^{-\int_{t_{k-1}}^{t_{k}}\left\langle n\left(m, h_{k-1}, \tau\right)\right\rangle d \tau / T} .
\end{aligned}
$$

The time-dependent Poisson parameter is the instantaneous mean photon number at the detector $\langle n(m, h, t)\rangle$ divided by the signal pulse duration $T$. For each $m$, we assume that the two interfering CFSK states are $\mid \alpha\left[\omega_{0}+\right.$ $(m-1) \Delta \omega,(m-1) \Delta \theta]\rangle$ and $\mid \beta\left[\omega_{0}+(h-1) \Delta \omega,(h-\right.$ 1) $\Delta \theta]\rangle$. We obtain

$$
\langle n(m, h, t)\rangle=2 \mathcal{T} n_{0}\{1-\cos [(h-m)(\Delta \omega t+\Delta \theta)]\},
$$

where $n_{0}$ is the mean photon number in the signal pulse and $\mathcal{T} \approx 1$ is the transmittance of the unbalanced beam splitter. Note that $\aleph$ depends on $|h-m|$, rather than on $m$ and $h$ separately.

To illustrate Bayesian inference with $M=4$ CFSK optimized for maximal sensitivity of the time-resolving quantum receiver, suppose that the initial state of the LO corresponds to hypothesis $h_{0}=1$, and the first photon is detected at $t_{1}$. We compute theoretical a posteriori Bayesian probabilities $\zeta_{t_{1}}(m)$ that the input signal is in state $m$ conditioned on the arrival time $t_{1}$ of the first photon by substituting $\aleph\left(t_{1}, 0,|1-m|\right)$ in (1). In Fig. 2 we show theoretical a posteriori Bayesian probabilities $\zeta_{t_{1}}(m)$ that the input signal is in state $m$ conditioned on the arrival time $t_{1}$ of the first photon. We see that probabilities $\zeta_{t_{1}}(2) \cdots \zeta_{t_{1}}(4)$ have a very distinct oscillatory dependence on photon detection time thanks to temporal fringing at 


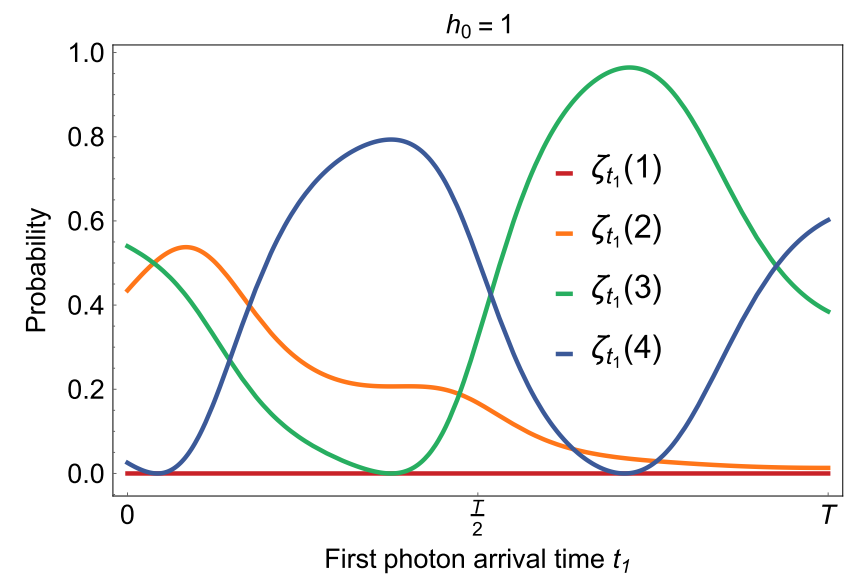

FIG. 2. The a posteriori Bayesian probabilities $\zeta_{t_{1}}(m)$ that the signal is in the state $m$ conditioned on the first photon detection at $t_{1}$ when the initial hypothesis is set to $h_{0}=1$. The $M=4$ CFSK modulation is optimized for maximum energy sensitivity of the receiver. $\Delta \theta=1.96, \Delta \omega T=\pi, n_{0}=2$, assuming ideal displacement and detector.

the detector. Therefore, the choice of $h_{1}$ strongly depends on the photon detection time. For the ideal displacement to vacuum the a posteriori probability for the hypothesis under test $\zeta_{t_{1}}(1) \equiv 0$. Different optimization goals can lead to different time dependences. The a posteriori Bayesian probabilities for legacy nonorthogonal protocols are monotonic, cf. Ref. [13], thus detection times do not influence the choice of a posterior hypothesis. Beyond this idealistic receiver model, Bayesian strategies can adapt to experimental imperfections. In practice, $\langle n(m, h, t)\rangle$ contains contributions due to imperfect interference, background, and dark counts.

The unique feature of this quantum receiver is that it adapts right after a photon detection at the SPD. All experimentally demonstrated receivers to date instead use predefined measurement stages of fixed duration and a decision-making tree. If we precomputed a decisionmaking tree, we would require $\mathrm{MT} / d t+(\mathrm{MT} / d t)^{2}+$ $\cdots+(\mathrm{MT} / d t)^{f}$ memory nodes, where $d t$ is the temporal resolution of the receiver and $f$ is the maximal number of photons that are expected during a signal pulse duration. Instead, we precompute and store values of $\aleph\left(t_{k}, t_{k-1},\left|h_{k-1}-m\right|\right)$, using just $M(T / d t)^{2} / 2$ memory nodes. For example, for $M=8$ and $T / d t=64$, we store 16384 precomputed values. When the SPD clicks, the photon detection time $t_{k}$ selects a Bayesian likelihood for each hypothesis using triangular $\left(t_{k}>t_{k-1}\right)$ probability matrices (Fig. 3). To further optimize computations, we normalize $\aleph\left(t_{k}, t_{k-1},\left|h_{k-1}-m\right|\right)$ such that $\max \left[\aleph\left(t_{k}, t_{k-1},\left|h_{k-1}-m\right|\right)\right]=1$. The bright areas seen in Fig. 3 correspond to a high likelihood of the particular hypothesis. Typically, for instance, it is likely that a click results in a change of the hypothesis. However, in
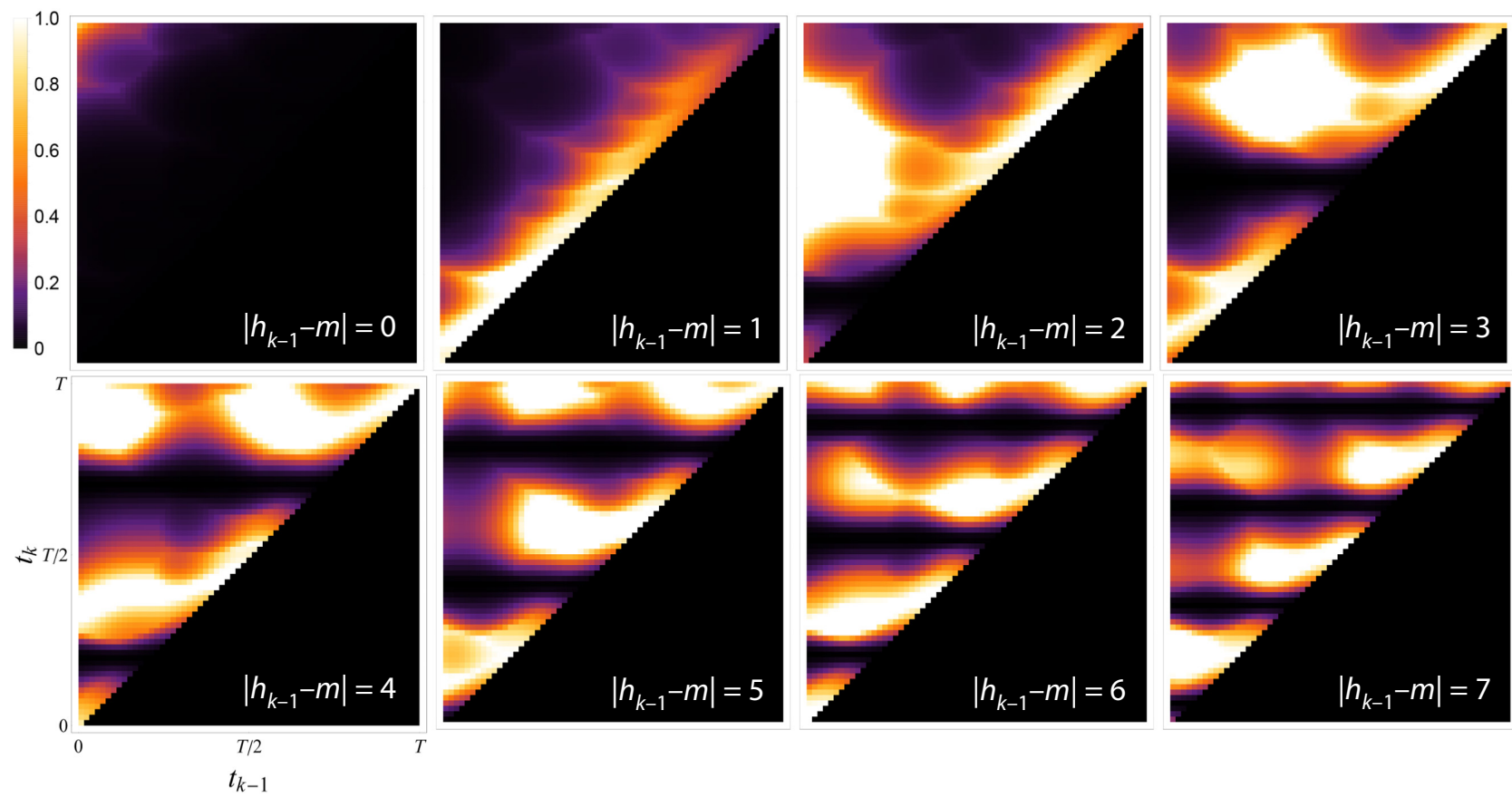

FIG. 3. The Bayesian likelihood $\aleph\left(t_{k}, t_{k-1},\left|h_{k-1}-m\right|\right)$ that the input state is $\left|\psi_{m}\right\rangle, m \in 1 . . M$ as a function of the current photon detection time $t_{k}$, previous photon detection time $t_{k-1}<t_{k}$, and the current state of the LO $h_{k-1}$, for an $M=8$ CFSK protocol. A photon detection at time $t_{k}$ leads to an update of posterior probabilities $\zeta_{t_{k}}(m)$ that the input state is $m$. To aid real-time calculations, we renormalize likelihood functions such that $\max \left[\aleph\left(t_{k}, t_{k-1},\left|h_{k-1}-m\right|\right)\right]=1$. 
a practical system with noise, detecting a photon may lead to no hypothesis change, particularly when the interval $t_{k}-t_{k-1}$ is large. This effect can be seen in Fig. 3 $\left(\left|h_{k-1}-m\right|=0\right)$, where low values of the likelihood function correspond to a hypothesis change. High values mean that the hypothesis should not be changed, and for our receiver this happens only when $t_{k-1} \gtrsim 0$ and $t_{k} \lesssim T$.

In stark contrast to detection schemes used in prior $M$-ary quantum state discrimination experiments [14,15], this time-resolved strategy allows testing of an unconstrained number of hypotheses $f$ and allocates the optimal time to probe each hypothesis. In addition, it alleviates the need for photon-number-resolved detection, cf. Ref. [15]. This strategy can be adopted for other nonorthogonal encoding protocols, such as phase-shift keying (PSK), quadrature amplitude modulation, etc. Theoretically, the time-resolved strategy is the most energy-sensitive detection method known to date for these communication protocols.

\section{EXPERIMENTAL IMPLEMENTATION}

Figure 4 shows the experimental setup to test the quantum receiver for the time-resolved coherent-state discrimination method. The continuous-wave laser at $633 \mathrm{~nm}$ is attenuated and sent via fiber to a 99:1 fiber beam splitter

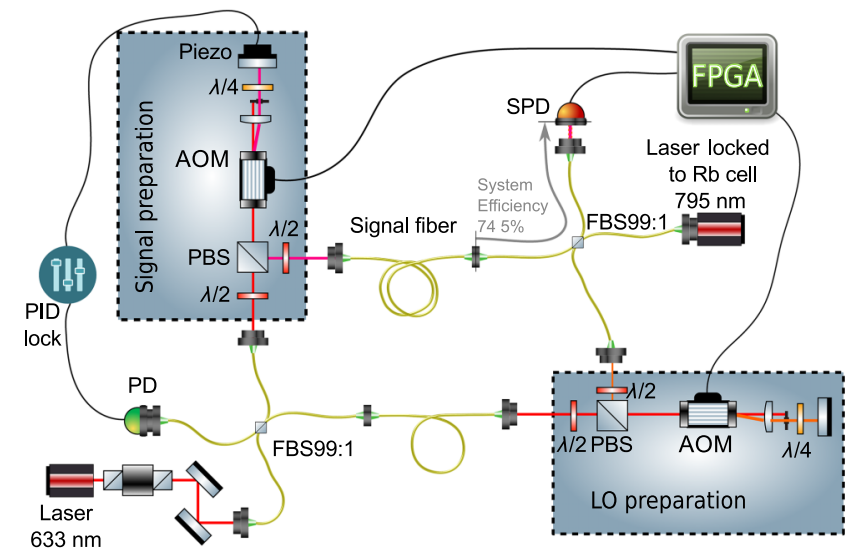

FIG. 4. Experimental implementation of an $M$-ary CFSK receiver. The light from a 633-nm laser is split 99:1 on an FBS and sent to the signal and LO preparation stages. We prepare CFSK and LO states by simultaneous frequency and phase modulation implemented with a double-pass AOM. The displacement is performed by interfering the input state with the $\mathrm{LO}$ on another 99:1 FBS. The displaced signal is sent to an SPD. The FPGA registers the times of photon detections, runs the discrimination algorithm and generates rf pulses to control the AOMs. The setup is interferometrically stabilized using a counter-propagating laser at $795 \mathrm{~nm}$, analog photodiode (PD), a piezo mirror, and a standalone lock (PID). PBS, polarization beam splitter; $\lambda / 2$ and $\lambda / 4$, half- and quarter-wave plates. The input of the quantum receiver is connected to the output of the signal fiber with a fiber mating sleeve.
(FBS) to generate the LO and the signal pulse, respectively. Each of the two beams undergoes state preparation with a double-pass acousto-optical modulator (AOM). AOMs are driven by controllable sine waves at approximately $80 \mathrm{MHz}$, synthesized by a digital-to-analog converter (DAC) controlled with a field programmable gate array (FPGA). Both the input signal and the LO are coupled back into the fiber. The signal at the fiber mating sleeve is defined as the input to the receiver, see Fig. 4. The receiver is comprised of LO and a 99:1 FBS for state displacement followed by an SPD, whose electronic output is connected to the FPGA for time tagging of the detection and Bayesian inference. To characterize the energy sensitivity of quantum state discrimination, we measure the system efficiency of the quantum receiver from the input. Optical losses amount to $11.4(5) \%$, and the SPD [22] detection efficiency is $84.0(3) \%$ (measured relative to a trap detector [23], see Appendix A), resulting in a system efficiency of $74.5(6) \%$.

The pair of 99:1 FBSs in Fig. 4 defines a Mach-Zehnder interferometer, whose relative path length is interferometrically stabilized. The stabilization is implemented with a counter-propagating laser at $795 \mathrm{~nm}$ frequency stabilized to a rubidium atomic line, a photodiode, and a piezo mirror controlled with stand-alone locking electronics. The signal from the SPD is used to set the locking point close to the minimum of the 633-nm fringe. To lock, the stabilization laser is switched on with a dedicated AOM and the FPGA sends identical frequencies to both the signal and LO arms of the Mach-Zehnder interferometer. To discriminate, the stabilization laser is switched off, and the FPGA executes the adaptive discrimination algorithm. The duty factor of the signal pulses is $\leq 50 \%$. The observed visibility of the interferometer reaches $99.7 \%$. To set the average number of photons per symbol in a signal pulse and the intensity of the LO, one of the two interferometer paths is blocked and the count rate on the SPD is measured.

We generate $M=4,6,8,12$, and 16 CFSK states. We adjust the CFSK parameters $\Delta \omega T$ and $\Delta \theta$ such that the quantum-measurement advantage maximizes energy sensitivity through numerical simulation, see Table I in Appendix B. In addition, we optimize the power of the LO for best error correction, not for the best displacement of the signal state to vacuum with the correct hypothesis, cf. Refs. $[13,16]$. The adjacent coherent states are shifted by $\Delta \omega=2 \pi \times 7629 \mathrm{rad} / \mathrm{s}$. The dimensionless detuning $\Delta \omega T$ is controlled by choosing the appropriate duration of signal pulses.

\section{RESULTS AND DISCUSSION}

We characterize our quantum receiver with $M=$ $4,6,8,12$, and 16 alphabets. Properties of our quantum receiver are illustrated using the $M=8$ case. Figure 5 shows the experimental error probability $P_{\mathrm{E}}$ in 

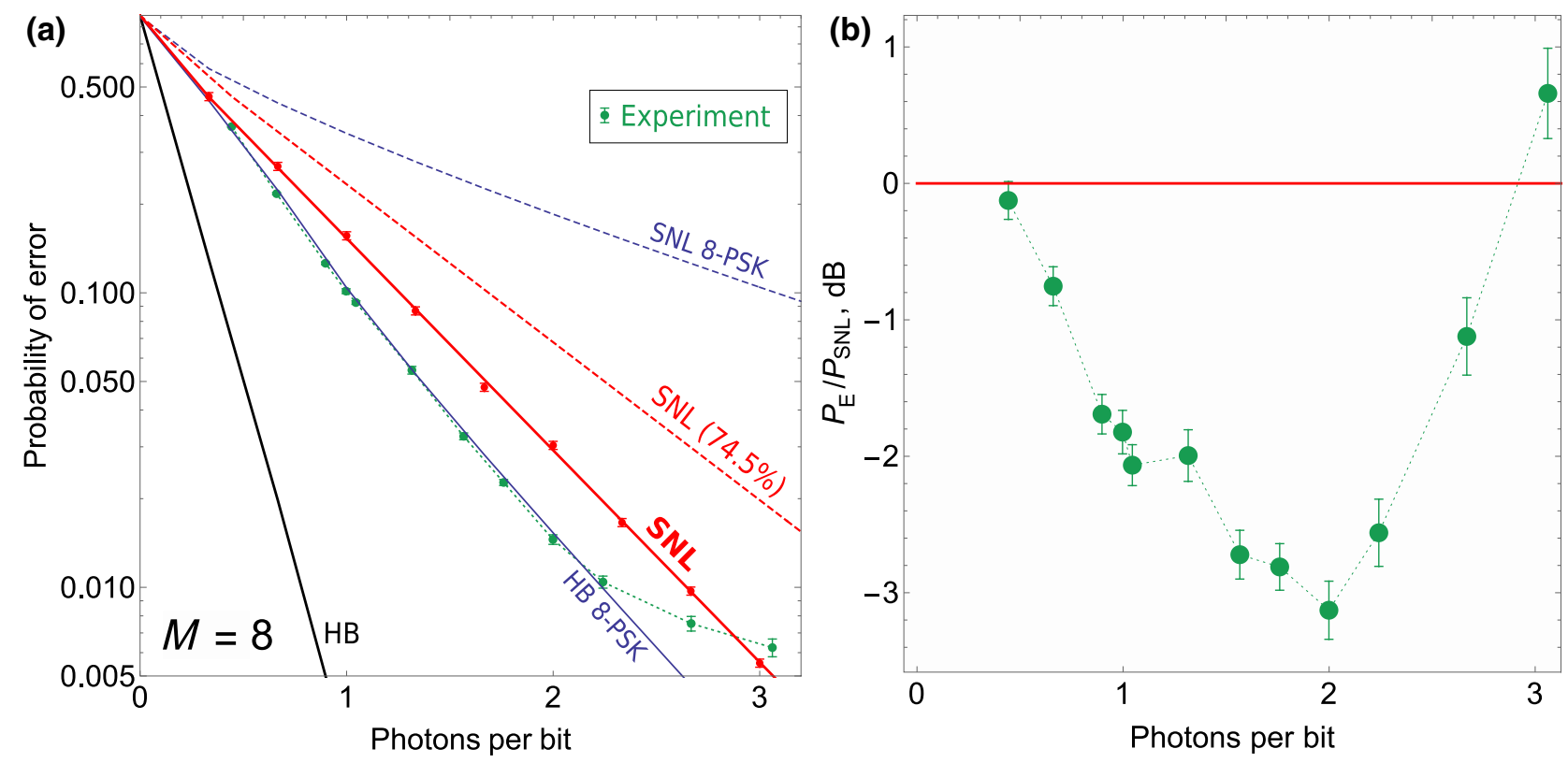

FIG. 5. (a) Measured performance (green points) below the SNL of the optimal classical receiver (solid red line) is unconditionally nonclassical. This is seen for a range of approximately 0.5 to approximately 2.5 photons/bit. For comparison, we also include the SNL adjusted to the same system efficiency as our receiver (dashed red line). Also shown is the HB and the HB of an 8-PSK protocol and the SNL for 8-PSK as indicated. (b) Measured sensitivity is plotted as a ratio to the SNL (red horizontal line). Error bars correspond to one standard deviation.

discriminating between eight CFSK states as a function of the mean photon number per bit, $n_{b}=n_{0} / \log _{2} M$ for the protocol optimized for maximum energy sensitivity, $\Delta \omega T=\pi \mathrm{rad}$ and $\Delta \theta=2.55 \mathrm{rad}$. Also shown is the SNL for the ideal classical detection, along with SNL scaled to our system efficiency of approximately $74.5 \%$, and the Helstrom bound (HB) [24].

We see that our receiver performs better than an ideal classical receiver for the input energies between approximately 0.5 and approximately 2.5 photons per bit with a maximum sensitivity improvement of 3.2(2) dB. In comparing our result to an optimal classical receiver with the same system efficiency, we observe $>6$-dB improvement. Because the CFSK protocol family includes the legacy PSK communication protocol as a special case, it is possible to use our unified framework to compare the energy sensitivities of the two. Notably, this demonstrates an unprecedentedly high energy sensitivity of a communication link. To this end, our measured error rate is lower than the quantum sensitivity limit or HB of an $M=8$ PSK protocol by more than one standard deviation between 1 and 2 photons per bit as seen in Fig. 5. The experimental sensitivity of our receiver saturates at higher input energies. This is because in our implementation the effective switching time after a photon detection is about $1 \mu \mathrm{s}$. This inactive time is mainly due to the speed of sound in the AOMs. The more photons are received during discrimination, the longer the total inactive time of our adaptive circuit is, contributing to the observed saturation in sensitivity. Error rate charts vs $n_{b}$ and comprehensive sensitivity comparisons for other alphabet lengths are presented in Appendix B.

A single-photon measurement naturally provides detection times for each photon, this information is fundamentally unavailable classically. Therefore, to fully access the quantum-measurement advantage, legacy encodings created for classical measurement are not optimal. This work features both the quantum receiver and a matching communication protocol and demonstrates its advantages. Unlike PSK, whose classical and quantum-enabled sensitivities rapidly decrease with $M$, a quantum measurement in conjunction with the CFSK can significantly reverse this trend. We present the dependence of the observed symbol error rate on the length of the alphabet keeping the energy per bit at $n_{b}=1$, Fig. 6 . All the observed error rates are below the corresponding SNLs. The observed energy sensitivity of our $M=4$ protocol at $n_{b} \approx 1$ is approximately $3 \mathrm{~dB}$ better than the best experimentally reported sensitivity for any $M>2$ quantum receiver. The observed dependence on $M$ is favorable to classical, and quantum theoretical limits of a PSK protocol. We note that our experimentally acquired discrimination errors for CFSK with $M \geq 8$ are lower than the corresponding quantum discrimination error bounds (Helstrom bounds) [24] of PSK. Particularly, the observed error rate for $M=12$ and 16 is more than $3 \mathrm{~dB}$ below the PSK HB for the same alphabet. The observed decrease of experimental sensitivity with $M$ can be partially explained by the switching time, whose significance increases with $M$, because longer alphabets may 


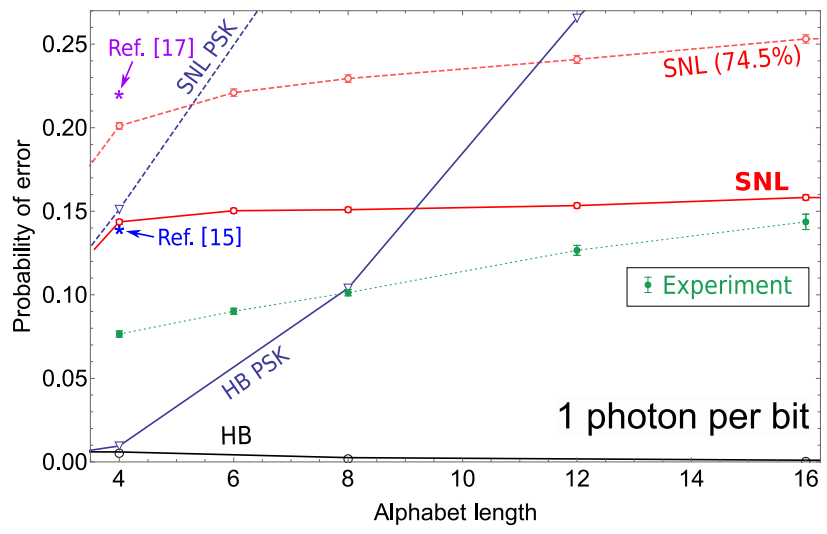

FIG. 6. The time-resolved receiver's scalability with $M$. The experimentally measured symbol error rate of the CFSK quantum receiver with the input of 1 photon/bit for protocols optimized for maximal energy sensitivity vs the alphabet length (green filled circles). Labeled are the ideal SNL, the SNL for a receiver with $74.5 \%$ system efficiency, and the HB, along with the SNL and HB of the PSK protocol. The best experimental sensitivity for the $M=4$ PSK protocol reported to date [15] _ blue star. Same for PPM [17] — purple star. Error bars correspond to one standard deviation. All connecting lines are guides for an eye.

require a larger number of photon detections for accurate discrimination.

As we show, switching to quantum receivers allows classical communication at the much lower power levels compatible with quantum networks and can eliminate the need for optical amplifiers. Therefore, our approach enables the coexistence of classical communication channels with entanglement-distribution and quantum-keydistribution channels in the same fiber, giving rise to a simple, unified quantum+classical network architecture.

\section{CONCLUSIONS}

To conclude, we experimentally study a telecommunication encoding designed from the ground up for quantum measurement of nonorthogonal coherent states. We build a time-resolving quantum $M \geq 4$ receiver with a record sensitivity at low signal energies, using off-the-shelf components. In addition, we assess the sensitivity scalability with the alphabet length $M$ for our communication protocol, and show that it scales favorably compared with legacy coherent protocols, such as PSK.

In general, quantum measurement can improve communication channel capacity beyond the classical limits. To this end, both the measurement scheme and a clever encoding play an important role. Our effort shows that quantum measurements can indeed offer valuable, heretofore unforeseen advantages for telecommunications leading to revolutionary improvements in channel resource use. The channel resource use offered by this work can be improved even further, up to the Holevo capacity limit [25]. This work can be used as a cornerstone for the development of even more efficient methods to communicate classically with the help of quantum measurements.

\section{ACKNOWLEDGMENTS}

We thank Michael Wayne, Alessandro Restelli, and Driss El Idrissi for their help with the FPGA at the initial stages of this work. We thank Emmanuel Knill, Sae Woo Nam, J. Javier Sabines Chesterking, Oliver Slattery, and Alan Migdall for a critical review of this manuscript and fruitful discussions. This work is partially supported by National Science Foundation through ECCS 1927674.

\section{APPENDIX A: SYSTEM-EFFICIENCY EVALUATION}

Our single-photon detector is a commercial avalanche photodiode-based photon counting module [22]. We calibrate its detection efficiency using a calibrated lighttrapping detector [23] with a detection efficiency of $98.5(1) \%$. We use the same light-trapping detector combined with commercial optical power meters to calibrate a set of optical attenuators. Attenuators are used to reduce the power of a HeNe laser beam to approximately $10^{5}$ photons per second to avoid saturation and nonlinearity at the SPD. The measured detection efficiency of the SPD is $84.0(3) \%$. We measure a total transmittance of $88.6(5) \%$ for the optical components of the receiver. The transmittance is obtained by measuring the optical power at the optical coupler of the SPD and dividing it by the optical power measured at the signal fiber output, see Fig. 3 of the paper. The optical power at the signal fiber output is

TABLE I. Parameters and performance of quantum $M$-ary CFSK receivers. Sensitivity comparison: lower values are better.

\begin{tabular}{lcccccc}
\hline \hline$M$ & $\begin{array}{c}\Delta \omega T, \\
\mathrm{rad}\end{array}$ & $\begin{array}{c}\Delta \theta, \\
\mathrm{rad}\end{array}$ & $\begin{array}{c}P_{\mathrm{E}} / P_{\mathrm{SNL}}^{\mathrm{CFSK}}, \\
\mathrm{dB}\end{array}$ & $\begin{array}{c}P_{\mathrm{E}} / P_{\mathrm{SNL}}^{\mathrm{CFSK}} 74.5 \%, \\
\mathrm{~dB}\end{array}$ & $\begin{array}{c}P_{\mathrm{E}} / P_{\mathrm{SNL}}^{\mathrm{PSK}}, \\
\mathrm{dB}\end{array}$ & $\begin{array}{c}P_{\mathrm{E}} / P_{\mathrm{HB}}^{\mathrm{PSK}}, \\
\mathrm{dB}\end{array}$ \\
\hline 4 & $\pi$ & 1.96 & $-7.3(4)$ & $-10.6(4)$ & $-7.6(4)$ & $\ldots$ \\
6 & $\pi$ & 2.35 & $-4.5(2)$ & $-8.1(3)$ & $-10.8(5)$ & $\ldots$ \\
8 & $\pi$ & 2.55 & $-3.1(2)$ & $-7.1(3)$ & $-12.2(3)$ & $-0.2(1)$ \\
12 & 4.22 & 1.67 & $-1.0(2)$ & $-3.7(2)$ & $-10.7(2)$ & $-4.6(2)$ \\
16 & $\pi$ & 2.55 & $-0.3(2)$ & $-2.6(2)$ & $-7.9(1)$ & $-5.2(1)$ \\
\hline \hline
\end{tabular}


adjusted to take into account a $-14.3-\mathrm{dB}$ Fresnel reflection at the fiber-to-air interface. The system efficiency of $74.5(6) \%$ is given by the product of optical transmittance and SPD detection efficiency.

\section{APPENDIX B: THE RECEIVER PERFORMANCE FOR $M=4,6,12$, AND 16}

We measure a discrimination error probability of CFSK quantum receivers for $M=4,6,12$, and 16 with the protocol parameters adjusted for maximal energy sensitivity, see Table I. Figure 7 shows the experimental error probability in discriminating between $M$-ary CFSK states as functions
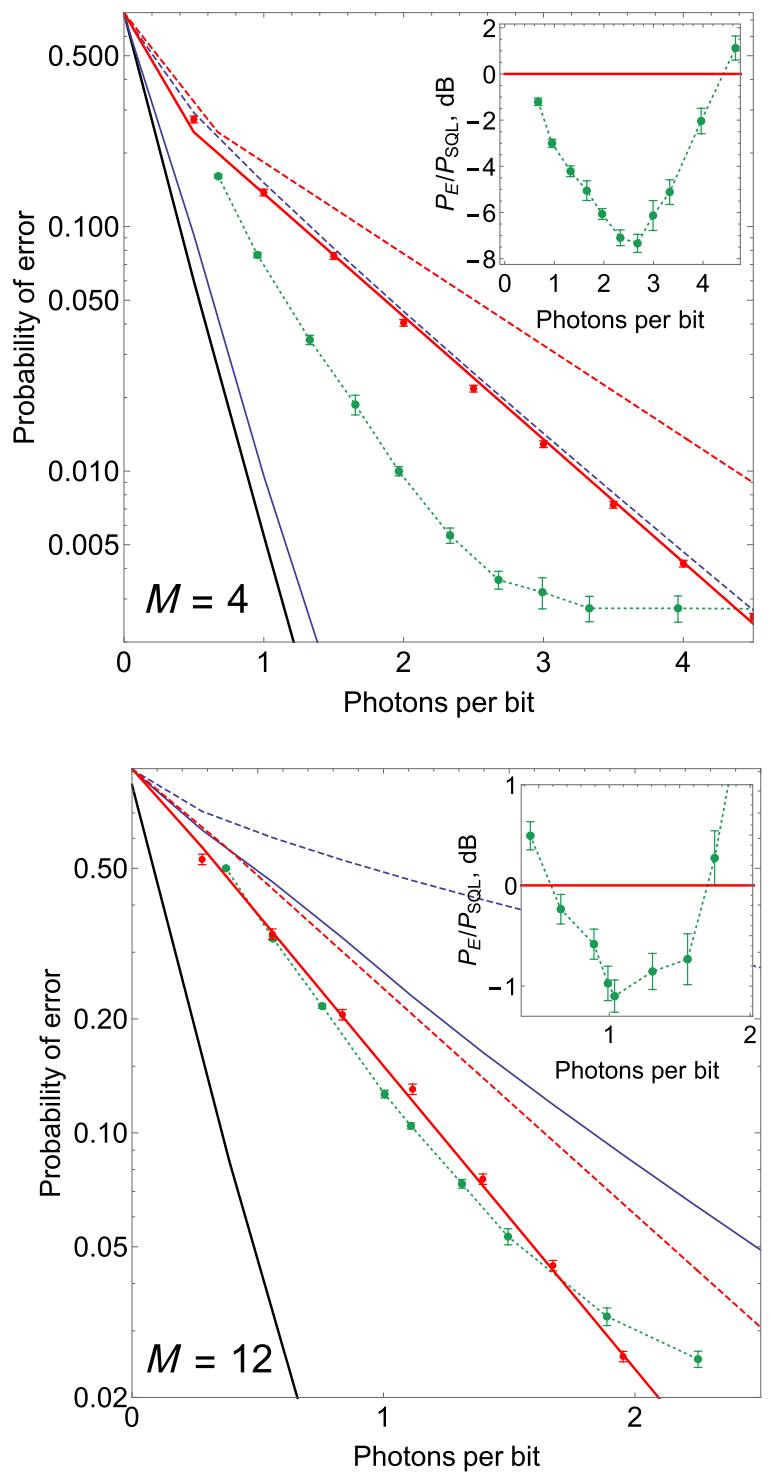

of the mean photon number per bit, $n_{b}=n_{0} / \log _{2} M$. Note that classical and nonclassical energy sensitivity limits of the PSK protocol saturate with $M$. For $M=8,12$, and 16 , the PSK HB is above the experimentally obtained values for our receiver. The comparison between the measured sensitivity and theoretical bounds is presented in Table I.

\section{APPENDIX C: $M$-ARY CFSK SHOT-NOISE LIMIT}

Consider an $M$-ary alphabet $\left\{\mathbf{s}_{m}\right\}$ of rectangular sinusoidal pulses defined for times $0<t<T$. For the $M$ ary CFSK, the frequency separation $\Delta \omega$ and the initial phase shift $\Delta \theta$ determine the degree to which one can
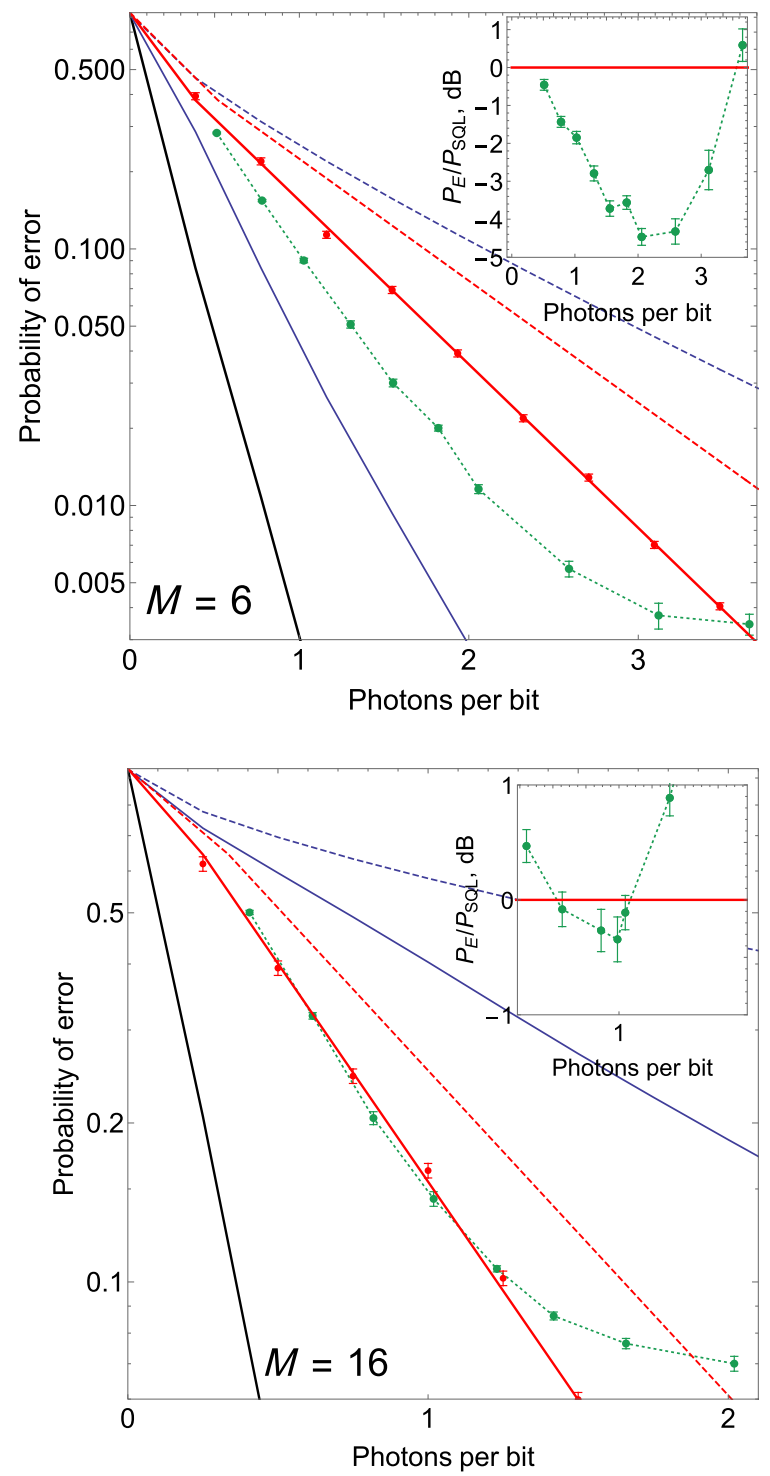

FIG. 7. Experimentally measured sensitivity of $M=4,6,12,16$ CFSK quantum receivers. The SNL is shown with a solid red line, the same classical limit adjusted to the experimental system efficiency of our receiver is shown with a dashed red line. The HB is shown with a solid black line. For further comparison, SNLs and HBs of corresponding $M$-ary PSK protocols are shown with solid and dashed blue lines, respectively. Insets: ratios of measured sensitivities to the SNL. Error bars correspond to one standard deviation statistical uncertainties. 
discriminate among the $M$ possible transmitted signals. We define correlation coefficients $\gamma_{m l}$ as a measure of the similarity (or dissimilarity) between a pair of signal waveforms:

$$
\begin{aligned}
\gamma_{m l} & =\mathbf{s}_{m} \cdot \mathbf{s}_{l} / \mathcal{E}_{s}=\int_{0}^{T} s_{m}(t) s_{l}(t) d t / \mathcal{E}_{s} \\
& =\cos [(m-l)(\Delta \omega T / 2+\Delta \theta)] \operatorname{sinc}[(m-l) \Delta \omega T / 2],
\end{aligned}
$$

where $\mathcal{E}_{s}=\int_{0}^{T} s_{m}^{2}(t) d t$ is the energy of a signal pulse. Note that all CFSK signals have the same energy.

The optimal classical receiver discriminates the input state by finding the largest cross-correlation between the received vector $\mathbf{r}$ and each of the $M$ nonorthogonal signal vectors $\left\{\mathbf{s}_{m}\right\}$ [19]:

$$
C\left(\mathbf{r}, \mathbf{s}_{m}\right)=\mathbf{r} \cdot \mathbf{s}_{m}, \quad m=1,2, \ldots, M
$$

For the transmitted signal $j$ and the communication channel corrupted by additive white Gaussian noise the received signal waveform can be written as

$$
r_{j}(t)=s_{j}(t)+n_{j}(t)
$$

where $n_{j}(t)$ is additive white Gaussian noise with a powerspectral density $N_{0} / 2 \mathrm{~W} / \mathrm{Hz}$. Thus, the cross-correlations read

$$
C\left(\mathbf{r}_{j}, \mathbf{s}_{m}\right)=\mathcal{E}_{s} \gamma_{j m}+\sqrt{\mathcal{E}_{s} N_{0} / 2} \tilde{n}_{j m}
$$

where $\tilde{n}_{j m}=\int_{0}^{T} n_{j}(t) s_{m}(t) d t / \sqrt{\mathcal{E}_{s} N_{0} / 2}$ is Gaussian noise with zero mean and unit variance. Note that noise components $\tilde{n}_{j m}$ of the CFSK symbols are not independent because of the nonzero correlations $\gamma_{m j}$. To simplify our numerical simulations, it is convenient to find an orthonormal basis $\{\boldsymbol{\phi}\}$ for $\left\{\mathbf{s}_{m}\right\}$, whose components are comprised of phase-coherent superpositions of the alphabet states, using a Gram-Schmidt orthogonalization procedure. In general, noise cannot be represented in the basis $\{\boldsymbol{\phi}\}$. However, we are only interested in the noise components that yield a nonzero correlation with $\left\{\mathbf{s}_{m}\right\}$. Therefore, for the purposes of calculating cross-correlations we write

$$
C\left(\mathbf{r}_{j}, \mathbf{s}_{m}\right)=\mathcal{E}_{s} \gamma_{j m}+\sqrt{\mathcal{E}_{s} N_{0} / 2} \sum_{i=1}^{M} s_{m i} n_{j i}
$$

where $s_{m i}=\int_{0}^{T} s_{m}(t) \phi_{i}(t) d t$ are the Gram-Schmidt coefficients for a set of signal vectors $\left\{\mathbf{s}_{m}\right\}$, and $n_{j i}$ are orthogonal (independent) components of the Gaussian noise with a zero-mean and unit variance.
When the only noise in the system is shot noise, one can write Eq. (C5) in a dimensionless form as

$$
C\left(\mathbf{r}_{j}, \mathbf{s}_{m}\right)=\sqrt{2\langle n\rangle} \gamma_{j m}+\sum_{i=1}^{M} s_{m i} n_{j i},
$$

where $\langle n\rangle$ is an average number of photons in a received signal.

To make a discrimination decision, one selects the $m$ that maximizes the correlation $C\left(\mathbf{r}_{j}, \mathbf{s}_{m}\right)$. To find the error probability of the optimal classical receiver in the shot-noise limit, we perform Monte Carlo simulations using coherent states' parameters of a CFSK alphabet for a range of input energies from 1 to 10 photons per symbol at the receiver and for CFSK parameters from Table I. We count errors in discrimination when the sent symbol $j$ is different from the received symbol $m\left(N_{j \neq m}\right)$ assuming equal probability for all symbols of the alphabet $j=1,2, \ldots, M$. Then, the best classically attainable discrimination probability can be approximated as

$$
P_{\mathrm{SNL}}^{\mathrm{CFSK}}=\frac{1}{M N} \sum_{j=1}^{M} N_{j \neq m},
$$

where $N$ is total number of trials. We perform the simulation until $\sum_{j=1}^{M} N_{j \neq m}$ reaches a certain value $N_{\text {set. }}$ The accuracy of this simulation is given by the standard deviation $\sqrt{N_{\text {set }}}$. These probabilities are presented in Figs. 5-7 as classical bounds with error bars at one standard deviation. Connecting lines that represent the SNL in Figs. 5 and 7 are based on linear fits of error probability logarithms.

[1] R. Landauer, Irreversibility and heat generation in the computing process, IBM J. Res. Dev. 5, 183 (1961).

[2] Antoine Bérut, Artak Arakelyan, Artyom Petrosyan, Sergio Ciliberto, Raoul Dillenschneider, and Eric Lutz, Experimental verification of landauer's principle linking information and thermodynamics, Nature 483, 187 (2012).

[3] Wojciech Hubert Zurek, Decoherence, einselection, and the quantum origins of the classical, Rev. Mod. Phys. 75, 715 (2003).

[4] R. S. Kennedy, Quantum limits in interferometric measurements, Research Laboratory of Electronics, MIT, Technical Report (1972).

[5] S. J. Dolinar, Quantum limits in interferometric measurements, Research Laboratory of Electronics, MIT, Technical Report (1973).

[6] Roy S. Bondurant, Near-quantum optimum receivers for the phase-quadrature coherent-state channel, Opt. Lett. 18, 1896 (1993).

[7] Kenji Tsujino, Daiji Fukuda, Go Fujii, Shuichiro Inoue, Mikio Fujiwara, Masahiro Takeoka, and Masahide Sasaki, 
Sub-shot-noise-limit discrimination of on-off keyed coherent signals via a quantum receiver with a superconducting transition edge sensor, Opt. Express 18, 8107 (2010).

[8] Saikat Guha, Jonathan L. Habif, and M. Takeoka, Approaching helstrom limits to optical pulse-position demodulation using single photon detection and optical feedback, J. Mod. Opt. 58, 257 (2011).

[9] Kenji Tsujino, Daiji Fukuda, Go Fujii, Shuichiro Inoue, Mikio Fujiwara, Masahiro Takeoka, and Masahide Sasaki, Quantum Receiver Beyond the Standard Quantum Limit of Coherent Optical Communication, Phys. Rev. Lett. 106, 250503 (2011).

[10] K. Kato, M. Osaki, M. Sasaki, and O. Hirota, Quantum detection and mutual information for qam and psk signals, IEEE T. Commun. 47, 248 (1999).

[11] F. E. Becerra, J. Fan, G. Baumgartner, S. V. Polyakov, J. Goldhar, J. T. Kosloski, and A. Migdall, $m$-ary-state phaseshift-keying discrimination below the homodyne limit, Phys. Rev. A 84, 062324 (2011).

[12] Yuan Zuo, Ke Li, and Bing Zhu, 16-QAM quantum receiver with hybrid structure outperforming the standard quantum limit, MATEC Web Conf. 61, 06008 (2016).

[13] C. R. Müller and Ch Marquardt, A robust quantum receiver for phase shift keyed signals, New J. Phys. 17, 032003 (2015).

[14] F. E. Becerra, J. Fan, G. Baumgartner, J. Goldhar, J. T. Kosloski, and A. Migdall, Experimental demonstration of a receiver beating the standard quantum limit for multiple nonorthogonal state discrimination, Nat. Photon 7, 147 (2013).

[15] F. E. Becerra, J. Fan, and A. Migdall, Photon number resolution enables quantum receiver for realistic coherent optical communications, Nat. Photon 9, 48 (2015).
[16] M. T. DiMario and F. E. Becerra, Robust Measurement for the Discrimination of Binary Coherent States, Phys. Rev. Lett. 121, 023603 (2018).

[17] Jian Chen, Jonathan L. Habif, Zachary Dutton, Richard Lazarus, and Saikat Guha, Optical codeword demodulation with error rates below the standard quantum limit using a conditional nulling receiver, Nat. Photonics 6, 374 (2012).

[18] I. A. Burenkov, O. V. Tikhonova, and S. V. Polyakov, Quantum receiver for large alphabet communication, Optica 5, 227 (2018).

[19] J. G. Proakis, Digital Communications, Electrical Engineering Series (Boston, MA, McGraw-Hill, 2001).

[20] H. Yuen, R. Kennedy, and M. Lax, Optimum testing of multiple hypotheses in quantum detection theory, IEEE Trans. Inf. Theory 21, 125 (1975).

[21] S. Ross, Introduction to Probability Models (New York, NY, Elsevier Science, 2007).

[22] PicoQuant, model Tau-SPAD-100, https://www.picoqu ant.com/products/category/photon-counting-detectors/tauspad-single-photon-counting-module. Please note that certain commercial equipment, instruments or materials are identified in this paper to foster understanding. Such identification does not imply recommendation or endorsement by the National Institute of Standards and Technology, nor does it imply that the materials or equipment identified are necessarily the best available for the purpose.

[23] N. P. Fox, Trap detectors and their properties, Metrologia 28, 197 (1991).

[24] Carl W. Helstrom, Quantum detection and estimation theory, J. Stat. Phys. 1, 231 (1969).

[25] A. S. Holevo, Capacity of a quantum communication channel, Probl. Peredachi Inform. (Problems Inform. Transm.) 15, 3 (1979). 